\title{
What can we learn from in ovo embryo vaccination?
}

\section{Introduction}

In ovo (in the egg) route of vaccine administration was first shown to be safe and effective in a 1982 publication for Marek's disease. ${ }^{1}$ Shortly after in 1992, in ovo vaccination becomes fully automated and has been commercially in wide use for over 25 years $^{2}$ for a number of poultry vaccines including Marek's disease, Infectious bursal disease, Newcastle disease, Avian influenza, Coccidiosis, and Mycoplasma gallisepticum..$^{2,3}$ Experimentally, an increasing number of publications have shown the safe and efficacious applicability of the route towards the administration of probiotics, growth promotors, hormones, or nutrients. ${ }^{2-4}$ Administration of biologics through the automated delivery system occurs around day $18 \pm 0.5$ of embryonic development (ED) through a dual needle: a piercing needle that pierce the shell over the air cell and an injection needle that goes through the piercing needle to a depth of about $2.5 \mathrm{~cm}$ delivering the biologics into the amniotic cavity. ${ }^{2,3}$ It takes 21 days for complete chick embryonic development. In ovo vaccination offers many advantages over conventional day-of-age vaccination. One of these advantages that is relevant to the topic raised here is its ability to stimulate an earlier innate and adaptive immune responses as compared with the conventional post-hatch (day 1 of age) vaccination. ${ }^{5}$ These earlier immune responses included the activation of certain immune cells or the differential expression and release of certain cytokines..$^{6-8}$

In chickens, fertilization of the ovulated ovum takes place within three hours of ovulation (as early as 15 minutes of ovulation) within the infundibulum, the first segment of the female reproductive tract. Fertilization is followed by cell division (or cleavage) of the fertilized egg. Embryonic cell division continues throughout the descent of the fertilized egg through the oviduct under body temperature of $40^{\circ} \mathrm{C}$. By the time of oviposition, the embryo (now forming a layer of cells called blastoderm) has about 50,000 cells (blastula stage) where the blastoderm remains at this stage due to the colder temperature (commercially is kept at $21-22^{\circ} \mathrm{C}$ ) outside the female reproductive tract. Once the blastoderm is placed under the influence of a higher temperature, be it natural or artificial incubation, the blastoderm is brought into action, thus continuing embryonic development. The blastoderm enters the next phase of embryonic development differentiating its cells into three germ layers (ectoderm, mesoderm, and endoderm) in a process called gastrulation where pluripotent stem cells migrate and make a decision that will determine their future fate. Of these stem cells, hematopoietic stem cells (HSC) become committed prior to colonizing hematopoietic organs. They originally emerge from the yolk sac (erythroid and a rare myeloid lineages), and the early aorta and the allantois (lineages with multipotent differentiation capacity). ${ }^{9-11}$ These cells later-on will populate primary immune organs for maturation and then leave to the peripheral including secondary lymphoid organs as functional immunocytes.

In ovo introductions of biologics were approached from the fact that chickens have developed certain immunologic functions before hatching. Thus, it is evident that the immune system of chick embryo is functional, albeit at a late stage of ED (last 3-4 days of ED). Obviously, there is an opportunity to modulate the component(s) of the chick immune system. Modulation could start very early during the first few days of ED. For instance, there is a potential to modulate the fate of HSC by administering modulators at very early stage of chick ED, possibly around day ED4. The effects of such modulator on
Volume 5 Issue 2 - 2018

\author{
Eid E Haddad \\ Department of Biological Sciences, Fayetteville State University, \\ USA \\ Correspondence: Eid E Haddad, Department of Biological \\ Sciences, Fayetteville State University, 1200 Murchison Road \\ Fayetteville, NC 2830,USA, Tel (910) 672 2436, Fax (910) 672 \\ II59,Email ehaddad@uncfsu.edu
}

Received: August 22, 2018 | Published: September 04, 2018

cell phenotype or its ability to express certain cytokines or even new ones is very possible, which may lead to fundamental changes in the field of immunology. Hormones (and non-replicating microorganisms) seem to be potential modulators since they are natural and were shown to play a role in the function of a mature immune system. ${ }^{12-15}$ Additionally, egg yolk, and to a certain degree egg white, contain various hormones including steroid and thyroid hormones. ${ }^{16}$ Another target day for in ovo administration of modulators could be around day ED9 which would potentiate the activity of immune components. Thus, in ovo vaccinations offer opportunities to new discoveries; immunology is a candidate field.

\section{Acknowledgements}

None.

\section{Conflict of interest}

The author declares there is no conflict of interest.

\section{References}

1. Sharma JM, Burmester BR. Resistance to Marek's disease at hatching in chickens vaccinated as embryos with the turkey herpesvirus. Avian Dis. 1982;26(1):134-49.

2. Ricks CA, Avakian A, Bryan T, et al. In ovo vaccination technology. Adv Vet Med. 1999;41:495-515.

3. Peebles ED. In ovo applications in poultry: A review. Poult Sci. 2018;97:(7):2322-2338.

4. Abdul-Cader MS, Palomino-Tapia V, Amarasinghe A, et al. Hatchery Vaccination Against Poultry Viral Diseases: Potential Mechanisms and Limitations. Viral Immunol. 2018;31(1):23-33.

5. Negash T, Al-Garib SO, Gruys E. Comparison of in ovo and post-hatch vaccination with particular reference to infectious bursal disease A review. Vet Q. 2004;26(2):76-87.

6. Gimeno IM, Faiz NM, Cortes AL, et al. In Ovo Vaccination with Turkey Herpesvirus Hastens Maturation of Chicken Embryo Immune Responses in Specific-Pathogen-Free Chickens. Avian Dis. 2015;59(3):375-83.

7. Abdul-Cader MS, Amarasinghe A, Palomino-Tapia V, et al. In ovo CpG DNA delivery increases innate and adaptive immune cells in respiratory, gastrointestinal and immune systems post-hatch correlating with lower infectious laryngotracheitis virus infection. PLoS One. 2018;13(3):e0193964. 
8. Viswanathan K, Rathish P, Gopinath VP, et al. In ovo delivery of Newcastle disease virus conjugated hybrid calcium phosphate nanoparticle and to study the cytokine profile induction. Mater Sci Eng C Mater Biol Appl. 2014;45:564-72.

9. Francoise-Lievre F. Commitment of hematopoietic stem cells in avian and mammalian embryos: an ongoing story. Int $J$ Dev Biol. 2005;49:(2-3):125-30.

10. Godin I, Cumano A. Of birds and mice: hematopoietic stem cell development. Int J Dev Biol. 2005;49:(2-3):251-7.

11. Jaffredo T, Bollerot K, Sugiyama D, et al. Tracing the hemangioblast during embryogenesis: developmental relationships between endothelial and hematopoietic cells. Int J Dev Biol. 2005;49(2-3):269-77.

12. Haddad EE, Mashaly MM. Effect of thyrotropin-releasing hormone, triiodothyronine, and chicken growth hormone on plasma concentrations of thyroxine, triiodothyronine, growth hormone, and growth of lymphoid organs and leukocyte populations in immature male chickens. Poult Sci. 1990;69(7):1094-102.
13. Haddad EE, Mashaly MM. In vivo effects of TRH, T3 and cGH on antibody production and $\mathrm{T}$ - and $\mathrm{B}$-lymphocytes proliferation in immature male chickens. Immunol Invest. 1991;20(7):557-68.

14. Haddad EE, Mashaly MM. Chicken growth hormone, triiodothyronine and thyrotropin releasing hormone modulation of the levels of chicken natural cell-mediated cytotoxicity. Dev Comp Immunol. 1991;15(1-2):65-71.

15. Haddad EE, Mashaly MM. Augmentation of natural cell-mediated cytotoxic activity by supernatant from in vitro mitogen-stimulated, in vivo hormone-treated lymphocytes in immature male (K strain) chickens. Immunol Invest. 1992;21:(4):365-75.

16. De Baere S, Rosendahl Larsen T, Devreese M, et al. Use of LC-MSMS as an alternative to currently available immunoassay methods to quantitate corticosterone in egg yolk and albumen. Anal Bioanal Chem. 2015;407(15):4351-62. 